\title{
Effects of Moderate Intensity Exercise on the Cortical Thickness and Subcortical Volumes of Preclinical Alzheimer's Disease Patients: A Pilot Study
}

\author{
Yoo Hyun Um¹, Sheng-Min Wang ${ }^{2}$, Nak-Young Kim², Dong Woo Kang ${ }^{3}$, \\ Hae-Ran $\mathrm{Na}^{2}$, Chang Uk Lee ${ }^{3}$, and Hyun Kook Lim ${ }^{2}$ \\ 1'Department of Psychiatry, St. Vincent's Hospital, College of Medicine, The Catholic University of Korea, Suwon, Republic of Korea \\ ${ }^{2}$ Department of Psychiatry, Yeouido St. Mary's Hospital, College of Medicine, The Catholic University of Korea, Seoul, Republic of Korea \\ ${ }^{3}$ Department of Psychiatry, Seoul St. Mary's Hospital, College of Medicine, The Catholic University of Korea, Seoul, Republic of Korea
}

Objective We aimed to explore the impact of moderate intensity exercise on the cortical thickness and subcortical volumes of preclinical Alzheimer's disease $(\mathrm{AD})$ patients.

Methods Sixty-three preclinical AD patients with magnetic resonance imaging (MRI) and 18-florbetaben positron emission tomography (PET) data were enrolled in the study. Information on demographic characteristics, cognitive battery scores, self-reported exercise habits were attained. Structural magnetic resonance images were analyzed and processed using Freesurfer v6.0.

Results Compared to Exercise group, Non-Exercise group demonstrated reduced cortical thickness in left parstriangularis, rostral middle frontal, entorhinal, superior frontal, lingual, superior parietal, lateral occipital, inferior parietal gyrus, temporal pole, precuneus, insula, fusiform gyrus, right precuneus, superiorparietal, lateral orbitofrontal, rostral middle frontal, medial orbitofrontal, superior frontal, lingual, middle temporal gyrus, insula, supramarginal, parahippocampal, paracentral gyrus. Volumes of right thalamus, caudate, putamen, pallidum, hippocampus, amygdala were also reduced in Non-Exercise group.

Conclusion Moderate intensity exercise affects cortical and subcortical structures in preclinical AD patients. Thus, physical exercise has a potential to be an effective intervention to prevent future cognitive decline in those at high risk of AD.

Psychiatry Investig 2020;17(6):613-619

Key Words Alzheimer's disease, Exercise, Cortical thickness, Subcortical volume, Structural MRI.

\section{INTRODUCTION}

Alzheimer's disease $(\mathrm{AD})$ is a major global health burden, with doubled world-wide prevalence in 26 years due to increase in aging population. ${ }^{1}$ Despite numerous efforts to unravel the exact mechanism of the disease, effective treatment and prevention options remain elusive. ${ }^{2}$ Instead of the single view of amyloid cascade hypothesis in understanding $\mathrm{AD}$, many researchers are beginning to advocate 'multifactorial hypothesis',

Received: June 5, 2020 Accepted: June 9, 2020

$\triangle$ Correspondence: Hyun Kook Lim, MD, PhD

Department of Psychiatry, Yeouido St. Mary's Hospital, College of Medicine, The Catholic University of Korea, 10 63-ro, Yeongdeungpo-gu, Seoul 07345, Republic of Korea

Tel: +82-2-3779-1048, Fax: +82-2-780-6577, E-mail: drblues@catholic.ac.kr (ac) This is an Open Access article distributed under the terms of the Creative Commons Attribution Non-Commercial License (https://creativecommons.org/licenses/bync/4.0) which permits unrestricted non-commercial use, distribution, and reproduction in any medium, provided the original work is properly cited. calling for the need to consider multiple targets when developing prevention or treatment strategies for $\mathrm{AD}^{3}{ }^{3} \mathrm{In}$ this regard, individual life style factors, environmental stress and resultant 'allostatic load' has been suggested as a major influencing factors in the disease trajectory of $\mathrm{AD}^{4}$ In line with this stance, recent major trials reported significant benefits of lifestyle interventions in those with high risk of $A D .^{5}$

Among many lifestyle and nonpharmacological interventions frequently discussed, ${ }^{6}$ physical exercise is increasingly accentuated as a potential therapeutic strategy for $\mathrm{AD},{ }^{7}$ with its beneficial effects on mitochondrial function, brain plasticity, neurogenesis and cerebral blood flow. ${ }^{7.8}$ One randomized controlled trial conducted to evaluate the effects of moderate-to high intensity aerobic exercise on mild AD patients demonstrated significantly reduced neuropsychiatric symptoms and cognition. ${ }^{9}$ Another randomized study on mild to moderate $\mathrm{AD}$ patients reported rather a contradicting result, demonstrating 
that a 4 month structured, moderate to high intensity aerobic exercise did not influence cognition, quality of life, or caregiver burden. ${ }^{10}$ According to recent systematic reviews, the impact of physical exercise on $\mathrm{AD}$ is inconclusive, due to lack of large sample sizes, methodological disparities across studies and lack of prospective designs. ${ }^{11,12}$ Moreover, studies on preclini$\mathrm{cal} \mathrm{AD}$ population remain relatively scarce.

Neuroimaging studies are also too scarce to demonstrate the impact of physical exercise on $\mathrm{AD}$ trajectory. There have been evidences of increased cortical thickness and attenuated influence of amyloid on cognition in older adults who have higher cardiorespiratory fitness, but sample sizes were small. ${ }^{13,14}$ There were no brain volume changes of $\mathrm{AD}$ patients after a 16-week aerobic exercise with duration of 60 minutes. ${ }^{15}$ Meanwhile, 12-week moderate intensity walking in mild cognitive impairment (MCI) patients was significantly associated with increased cortical thickness. However, the study lacked a modest sample size and information on amyloid deposition. ${ }^{16}$ Another study reported similar results, with 12-week multimodal physical exercise program only proven to be effective for MCI patients but not in $\mathrm{AD}$ patients. ${ }^{17}$ Contradicting results on $\mathrm{AD}$ and MCI patients may be due to the differential impact of exercise in the different stages of $\mathrm{AD}$ trajectory.

In this study, we aimed demonstrate the impact of physical exercise on the cortical thickness and subcortical volume of preclinical $\mathrm{AD}$ patients, who are cognitively normal but with amyloid deposition. We hypothesized that cortical thickness and subcortical volumes of preclinical dementia patients with physical exercise will be reduced when compared with their counterparts.

\section{METHODS}

\section{Subjects}

Sixty-three preclinical AD subjects were included in this study. They were recruited from the normal control volunteers of the Catholic Dementia Brain Imaging Database, which holds brain scans of outpatients and inpatients at the Department of Geriatric Psychiatry, the Saint Vincent's Hospital, the Catholic University of Korea from 2010 to 2016. The inclusion criteria of the Exercise group were as follows: 1) subjects aged $\geq 60$ years; 2) Mini-Mental Status Examination score $\geq 27$; 3) Clinical Dementia Rating $=0 ;{ }^{18} 4$ ) amyloid retention corresponds to global mean standard value uptake ratio (SUVR) $\geq 1.4$. 5) Doing moderate intensity aerobic exercise (e.g., mountain climbing, dancing, and swimming) more than one hour per day, 5 days per weeks. ${ }^{19}$ Most of the inclusion criteria of the Non-Exercise group were the same as the Exercise group [1)-4)], except for the criteria that the Non-Exercise group had a history of doing walking less than $10 \mathrm{~min}$ or no exercise per day, 5 days per weeks were included. ${ }^{19}$ The cognitive functions of the subjects were evaluated with the Korean version of Consortium to Establish a Registry for Alzheimer's Disease (CERAD-K), ${ }^{20}$ which assess the following cognitive domains: verbal fluency, 15-item Boston naming test (BNT), the Korean version of Mini Mental Status Examination (MMSE-K), ${ }^{21}$ constructional praxis (CP), word list memory (WLM), word list recall (WLR), word list recognition (WLRc), constructional recall (CR). The study was conducted in accordance with the ethical and safety guidelines set forth by the local Institutional Review Board of the Catholic University of Korea and written informed consent was obtained from all study subjects.

\section{PET acquisition}

FBB [(F-18) florbetaben] was produced and FBB-PET data were collected and analyzed as previously described. ${ }^{22}$ Each individual participant's MRI was utilized for co-registration and defining the ROI and for correcting partial volume effects from expanding cerebrospinal spaces accompanying cerebral atrophy. ${ }^{23,24}$ Analysis of the FBB PET data utilized a standardized uptake value ratio (SUVR) 90 min post-injection, using the cerebellar cortex region of interest as the reference. Global $\mathrm{A} \beta$ burden was expressed as the average SUVR of the mean for the following 5 cortical ROIs: frontal, superior parietal, lateral temporal, and anterior and posterior cingulate cortex/precuneus as described in previous study. ${ }^{24}$

The FBB PET data were acquired within 4 weeks of clinical screening and cognitive function test. We used a cut-off for 'high' or 'low' neocortical SUVR of 1.4, consistent with cut-off values used in previous FBB-PET study. ${ }^{25}$

\section{MRI acquisition}

Imaging data were collected at the Department of Radiology, St Vincent's Hospital, The Catholic University of Korea, using a 3T Siemens Verio machine and eight channel Siemens head coil (Siemens Medical Solutions, Erlangen, Germany). The parameters used for the T1-weighted volumetric magnetization-prepared rapid gradient echo scan sequences were $\mathrm{TE}=2.5 \mathrm{~ms}, \mathrm{TR}=1,900 \mathrm{~ms}$, inversion time $=900 \mathrm{~ms}, \mathrm{FOV}=250$ $\mathrm{mm}$, matrix $=256 \times 256$, and voxel size $=1.0 \times 1.0 \times 1.0 \mathrm{~mm}^{3}$

\section{Data analysis}

For cortical reconstruction and volumetric segmentation of the whole brain, Freesurfer image analysis suite (version 6.0, http://surfer.nmr.mgh.harvard.edu), which is documented and freely available online, was used. The technical details of these procedures have been described in previous publications. ${ }^{26,27}$ Briefly, the processing stream includes a Talairach transform of each subject's native brain, removal of the non-brain tissue, and segmentation of the gray matter/white matter (GM/WM) 
tissue. The cortical surface of each hemisphere was inflated to an average spherical surface to locate both the pial surface and the GM/WM boundary. The entire cortex of each subject was visually inspected, and any topological defects were corrected manually, blind to the subject's identity. The cortical thickness was computed as the shortest distance between the pial surface and the GM/WM boundary at each point across the cortical mantle. The global mean cortical thickness for each subject was computed by averaging the cortical thickness at each vertex, right and left hemispheres separately, and was used in the statistical analyses. The regional thickness value at each vertex for each subject was mapped to the surface of an average brain template allowing visualization of data across the entire cortical surface (described at http://surfer. nmr.mgh.harvard. edu/fswiki/FsAverage). In addition, the entire cerebral cortex was parcellated into 34 regions, ${ }^{28,29}$ and a variety of surface-based data, including maps of cortical volume and surface area as well as curvature and sulcal depth, were created. Data were resampled for all subjects onto a common spherical coordinate system. ${ }^{30}$ The cortical map of each subject was smoothed with a Gaussian kernel of 10-mm full width at half-maximum for the entire cortex analyses. The subcortical volumes were obtained from the automated procedure for volumetric measures of the brain structures implemented in Freesurfer. In all, 27 volumetric measures were investigated and extracted seven subcortical structures (white matter, caudate, thalamus, pallidum, putamen, hippocampus, and amygdala) from each hemisphere.

\section{Statistical analysis}

Statistical analyses for demographic data were performed with the Statistical Package for Social Sciences software (SPSS, version 20.0, IBM Corp., Armonk, NY, USA). Assumptions for normality were tested for all continuous variables. Normality was tested using the Kolmogorov-Smirnov test. All variables were normally distributed. The independent t-test and the $\chi^{2}$ test were used to assess potential differences between the Exercise groups and Non exercise groups for all demographic variables. All statistical analyses had a two-tailed a level of $<0.05$ for defining statistical significance. The general linear model (GLM) was implemented at each vertex in the whole brain to identify the brain regions in which the Exercise groups showed significant differences in cortical thickness relative to Non exercise group, using the FreeSurfer's mri_glmfit (described at http://surfer.nmr.mgh.harvard. edu/fswiki/mri_glmfit). The All analyses were performed for the right and left hemispheres separately. The threshold was set at $\mathrm{p}<0.05$ [false discovery rate (FDR)] to resolve the problem of multiple comparisons. The seven subcortical structure volumes (i.e., total white matter volumes, thalamus, caudate nucleus, putamen, pallidum, hippocampus, and amygdala) were imported into the SPSS
20.0 software for statistical analyses (IBM Corp.). To assess the main effects of diagnosis on the volume of subcortical structures, we used analysis of covariance (ANCOVA) with TIV, education, gender, and age as nuisance variables.

\section{RESULTS}

Demographic and clinical characteristics of the study participants are summarized in Table 1. There was no significant difference in age, education, gender and CDR scores between Exercise group and Non-Exercise group. Among the cognitive domains of CERAD-K, there were significant differences in WLM, WLR, WRLc, and CR scores between the two groups, with Non Exercise group concordantly displaying lower scores.

When compared with Exercise group, Non-Exercise group

Table 1. Demographic and clinical characteristics of the study participants

\begin{tabular}{|c|c|c|c|}
\hline & $\begin{array}{l}\text { Exercise group } \\
\qquad(\mathrm{N}=33)\end{array}$ & $\begin{array}{l}\text { Non Exercise } \\
\text { group }(\mathrm{N}=30)\end{array}$ & $\mathrm{p}$ value \\
\hline Age (years $\pm \mathrm{SD})$ & $70.1 \pm 8.1$ & $70.2 \pm 7.9$ & NS \\
\hline Education (years $\pm S D$ ) & $11.2 \pm 4.8$ & $10.4 \pm 5.1$ & NS \\
\hline $\operatorname{Sex}(M: F)$ & $12: 21$ & $13: 17$ & NS \\
\hline CDR (SD) & 0 & 0 & \\
\hline SUVR (SD) & & & NS \\
\hline ACC & $1.48 \pm 0.12$ & $1.42 \pm 0.21$ & NS \\
\hline FRC & $1.34 \pm 0.13$ & $1.32 \pm 0.12$ & NS \\
\hline PAR & $1.12 \pm 0.08$ & $1.24 \pm 0.10$ & NS \\
\hline LT & $1.40 \pm 0.11$ & $1.34 \pm 0.10$ & NS \\
\hline PRC & $1.44 \pm 0.12$ & $1.43 \pm 0.12$ & NS \\
\hline PCC & $1.62 \pm 0.15$ & $1.64 \pm 0.11$ & NS \\
\hline Mean & $1.50 \pm 0.12$ & $1.48 \pm 0.10$ & NS \\
\hline \multicolumn{4}{|l|}{ CERAD-K battery (SD) } \\
\hline VF & $13.4 \pm 3.9$ & $11.8 \pm 4.1$ & NS \\
\hline $\mathrm{BNT}$ & $10.9 \pm 2.7$ & $10.0 \pm 2.7$ & NS \\
\hline MMSE & $27.9 \pm 2.7$ & $27.2 \pm 2.3$ & NS \\
\hline WLM & $16.9 \pm 4.3$ & $13.7 \pm 4.7$ & 0.004 \\
\hline $\mathrm{CP}$ & $10.2 \pm 1.1$ & $10.4 \pm 1.1$ & NS \\
\hline WLR & $4.5 \pm 2.2$ & $2.3 \pm 2.4$ & $<0.001$ \\
\hline WLRc & $8.0 \pm 2.2$ & $5.9 \pm 2.7$ & $<0.001$ \\
\hline CR & $5.3 \pm 3.3$ & $2.6 \pm 2.2$ & $<0.001$ \\
\hline
\end{tabular}

SD: standard deviation, NS: not significant, CDR: Clinical Dementia Rating, SUVR: standard value uptake ratio, ACC: anterior cingulate, FRC: frontal cortex, PAR: parietal cortex, LT: lateral temporal cortex, PRC: precuneus, PCC: posterior cingulate, CERAD-K: the Korean version of Consortium to Establish a Registry for Alzheimer's disease, VF: verbal fluency; BNT: 15-item Boston Naming Test, MMSE: Mini Mental Status Examination, WLM: word list memory, CP: constructional praxis, WLR: word list recall, WLRc: word list recognition, CR: constructional recall 
demonstrated significantly reduced cortical thickness in left parstriangularis, rostral middle frontal, entorhinal, superior frontal, lingual, superior parietal, lateral occipital, inferior parietal gyrus, temporal pole, precuneus, insula, and fusiform gyrus (Table 2, Figure 1). Moreover, reduced cortical thickness in right precuneus, superiorparietal, lateral orbitofrontal, rostral middle frontal, medial orbitofrontal, superior frontal, lingual, middle temporal gyrus, insula, supramarginal, parahippocampal, paracentral gyrus was noted in Non-Exercise group (Table 2, Figure 1).

As for subcortical volumes, reduced subcortical volumes were noted in left thalamus, putamen, pallidum, hippocampus, amygdala in Non-Exercise group when compared with Exercise group (Table 3). Volumes of right thalamus, caudate, putamen, pallidum, hippocampus, amygdala were also reduced in Non-Exercise group (Table 3).

\section{DISCUSSION}

To the best of our knowledge, this is the first study to explore the effects of physical exercise on cortical thickness of preclinical AD patients. According to our results, WLM, WLR, WRLc and CR scores were reduced in Non-Exercise group. Moreover, cortical thickness, subcortical volumes of certain brain regions were reduced in Non-Exercise group when compared with Exercise group.

Reasons for the disparities in the CERAD-K scores between the two groups may be attributable to the benevolent effects of moderate-to high intensity exercise in daily life. Our results are in line with previous results, where there was a dose-response relationship between the amount of physical activity and cognitive function among the elderly. ${ }^{31}$ Moreover, across the lifespan, subjects who reported that they have been phys-

Table 2. Voxel wise group comparison results where a significant cortical thinning was observed in Non-Exercise group relative to Exercise group (FDR corrected, $p<0.05$ )

\begin{tabular}{|c|c|c|c|c|c|c|}
\hline \multirow{2}{*}{ Region } & \multirow{2}{*}{ Cluster size $\left(\mathrm{mm}^{2}\right)$} & \multirow{2}{*}{ Number of vertex } & \multirow{2}{*}{$\mathrm{T} \max$} & \multicolumn{3}{|c|}{ Talairach coordinates } \\
\hline & & & & $\mathrm{X}$ & $\mathrm{Y}$ & $\mathrm{Z}$ \\
\hline \multicolumn{7}{|l|}{ Left } \\
\hline Parstriangularis & 184.75 & 264 & 5.836 & -48.2 & 33.3 & 1.5 \\
\hline Rostral middle frontal & 192.58 & 346 & 5.714 & -41.0 & 31.1 & 19.4 \\
\hline Entorhinal & 162.01 & 348 & 4.565 & -32.2 & -9.8 & -31.8 \\
\hline Superior frontal & 112.62 & 183 & 4.041 & -10.4 & 29.8 & 30.7 \\
\hline Lingual & 101.41 & 215 & 3.875 & -26.2 & -45.6 & -6.2 \\
\hline Superior parietal & 29.77 & 80 & 3.741 & -27.6 & -60.8 & 44.1 \\
\hline Lateral occipital & 44.07 & 80 & 3.721 & -42.2 & -68.1 & 6.5 \\
\hline Inferior parietal & 59.10 & 102 & 3.707 & -39.6 & -79.3 & 15.2 \\
\hline Temporal pole & 77.58 & 91 & 3.559 & -38.4 & 8.7 & -36.9 \\
\hline Precuneus & 125.61 & 236 & 3.537 & -18.2 & -71.4 & 30.4 \\
\hline Insula & 34.88 & 101 & 3.512 & -37.4 & -1.1 & -14.4 \\
\hline Fusiform & 34.75 & 61 & 3.501 & -32.7 & -36.6 & -21.6 \\
\hline \multicolumn{7}{|l|}{ Right } \\
\hline Precuneus & 58.37 & 164 & 5.254 & 7.2 & -51.5 & 62.7 \\
\hline Superiorparietal & 82.11 & 134 & 4.622 & 18.7 & -78.4 & 41.6 \\
\hline Lateral orbitofrontal & 290.44 & 573 & 4.406 & 37.2 & 28.2 & -16.6 \\
\hline Rostral middle frontal & 412.33 & 575 & 4.224 & 30.6 & 49.5 & 2.5 \\
\hline Medial orbitofrontal & 151.07 & 271 & 4.189 & 12.6 & 43.9 & -4.6 \\
\hline Superior frontal & 62.55 & 123 & 3.925 & 10.4 & 29.3 & 33.7 \\
\hline Lingual & 67.47 & 166 & 3.636 & 23.3 & -53.1 & -2.2 \\
\hline Middle temporal & 34.27 & 56 & 3.623 & 54.4 & -17.3 & -24.2 \\
\hline Insula & 22.02 & 82 & 3.605 & 32.7 & -24.8 & 19.1 \\
\hline Supramarginal & 33.98 & 70 & 3.456 & 55.4 & -37.0 & 19.1 \\
\hline Parahippocampal & 21.11 & 72 & 3.328 & 21.6 & -14.8 & -27.8 \\
\hline Paracentral & 47.66 & 131 & 3.279 & 13.9 & -24.6 & 46.3 \\
\hline
\end{tabular}

FDR: false discovery rate 


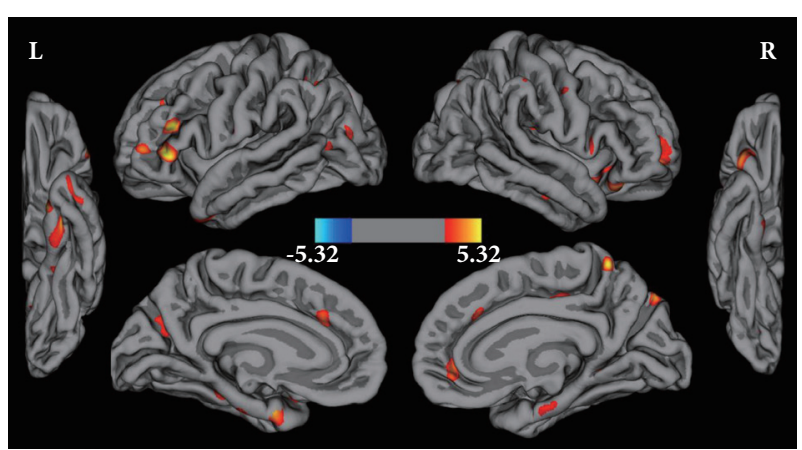

Figure 1. Statistical maps corrected for age, education, and gender showing increased cortical thickness in the Exercise group relative to the Non-exercise group ( $p<0.05$ FDR corrected). FDR: false discovery rate.

Table 3. Group analysis results of subcortical volumes

\begin{tabular}{lccc}
\hline $\begin{array}{c}\text { Region } \\
\left(\mathrm{mm}^{3} \pm \mathrm{SD}\right)\end{array}$ & $\begin{array}{c}\text { Exercise group } \\
(\mathrm{N}=33)\end{array}$ & $\begin{array}{c}\text { Non-exercise } \\
\text { group } \\
(\mathrm{N}=30)\end{array}$ & p value \\
\hline $\begin{array}{c}\text { Left } \\
\text { Thalamus }\end{array}$ & $6,697 \pm 666$ & $6,286 \pm 421$ & 0.004 \\
Caudate & $3,242 \pm 450$ & $3,129 \pm 463$ & 0.301 \\
Putamen & $4,221 \pm 445$ & $3,910 \pm 503$ & 0.007 \\
Pallidum & $1,884 \pm 197$ & $1,768 \pm 202$ & 0.016 \\
Hippocampus & $3,635 \pm 367$ & $2,972 \pm 346$ & $<0.001$ \\
Amygdala & $1,389 \pm 182$ & $1,139 \pm 152$ & $<0.001$ \\
Right & & & \\
Thalamus & $6,669 \pm 665$ & $6,230 \pm 415$ & 0.002 \\
Caudate & $3,275 \pm 496$ & $3,108 \pm 361$ & 0.117 \\
Putamen & $4,328 \pm 541$ & $4,012 \pm 387$ & 0.007 \\
Pallidum & $1,816 \pm 208$ & $1,769 \pm 199$ & 0.332 \\
Hippocampus & $3,826 \pm 463$ & $3,227 \pm 468$ & $<0.001$ \\
Amygdala & $1,573 \pm 224$ & $1,322 \pm 206$ & $<0.001$ \\
\hline
\end{tabular}

SD: standard deviation

ically active both in the early adulthood and mid-to-late adulthood were at lower risk of subjective cognitive decline. ${ }^{32} \mathrm{An}$ other study on the sample from the national survey reported that in those with family history of $\mathrm{AD}$, subjective reports of higher physical activity was associated with greater cognitive function. ${ }^{33}$ Even higher 'perceived' physical activity in subjective reports were associated with increased cognitive ability. ${ }^{34}$ Meanwhile, Non-Exercise group in our study demonstrated lower scores in WLM, WLR, WRLc, and CR scores of the CERAD-K battery, which are closely related to the episodic memory assessment. They are domains where AD patients typically score less than their counterparts. Our results could imply that effects of exercise directly influence cognitive domains that are typically attacked by $\mathrm{AD}$. Biological mechanisms explaining how exercise beneficially affects cognitive function are scarce. Many predict that exercise-induced elevations in physical func- tion in turn results in cognitive function. ${ }^{35}$ This is thought to be mediated by increased serum levels of brain-derived neurotropic factors (BDNF), insulin-like growth factor type-1 (IGF-1) and vascular endothelial growth factors (VEGF) often noted after physical exercise. ${ }^{36}$

The aforementioned increased markers of neurogenesis may explain the disparities in the cortical thickness between Exercise and Non-Exercise group in our study. Indeed, one study on healthy elders found that BDNF elevation was higher after physical exercise when compared with those measured after cognitive training. ${ }^{37}$ Another explanation for the different results of cortical thickness between the two groups is the protective effects of exercise on neurovascular function. Exercise significantly improved blood brain barrier integrity in elderly women, ${ }^{38}$ and exercise significantly increased endothelial function and cerebral blood flow, which can prevent neuronal hypoxia and degeneration. ${ }^{39,40}$ Meanwhile, many of the regions that showed reduced cortical thickness in Non-Exercise group are components of default mode network (DMN), encompassing posterior cingulate cortex, precuneus, medial prefrontal cortex, mesial and inferior temporal cortex and inferior parietal cortex. ${ }^{41}$ It is significant that important hubs of DMN, bilateral precuneus were all affected in None-Exercise group. It is in with a previous study where increased functional connectivity of DMN was observed in MCI patients after 12-week aerobic exercise. ${ }^{42} \mathrm{DMN}$ is a well-known for its vulnerability to disruption due to incipient $\mathrm{AD}$ pathology, and our results imply the preventive effects of physical exercise may be exerted through maintaining structural components of DMN in preclinical $\mathrm{AD}$ patients.

As for the results on subcortical volumes of the two groups, non-exercise group demonstrated reduced volumes of thalamus, caudate, putamen, pallidum, hippocampus, amygdala. Thalamic volume loss was proposed to be an early indicator of poorer cognitive performance in amnestic MCI patients in one study, ${ }^{43}$ and early involvement of thalamus and striatum were noted in familial $\mathrm{AD}$ patients. ${ }^{44}$ Moreover, subcortical volume loss increased the risk of conversion from $\mathrm{MCI}$ to $\mathrm{AD}{ }^{45}$ Subcortical structures are proposed to be involved in the early stages of $\mathrm{AD}$ trajectory. In this regard, how exercise impacts those in the incipient stage of $\mathrm{AD}$ trajectory is of critical issue, but relevant studies are still scarce. A Japanese study on healthy elders demonstrated a meaningful result, with larger volumes of left hippocampus and bilateral nucleus accumbens in those exposed to frequent exercise habits. ${ }^{46}$ Our study results are in line with the aforementioned result, and disparities in the subcortical volumes of the two groups in our study suggest the beneficial effects of exercise not only impact cortical structures, but also core subcortical structures in preclinical $\mathrm{AD}$ patients.

There are several limitations that must be taken into con- 
sideration. First, subjects were recruited from a single center, which limits generalizability of results. Second, sample sizes were small. Third, assessment of quantity and intensity of exercise were solely based on patients' report, which is subject to recall bias. Fourth, the study was a retrospective, cross-sectional design. Fourth, information on apolipoprotein E (APOE) genotype was not provided, which limits more detailed interpretation of interactive effect of APOE and exercise on patients at high risk of $\mathrm{AD}$.

There have been numerous interventional studies trying to explore the effects of exercise on cognition. ${ }^{47-50}$ However, many are inconclusive due to heterogeneity of the study design and methods. Future studies should consider well-controlled and stringent selection of study population, structured, uniform exercise protocol and adequate sample sizes to achieve more conclusive findings on the relationship between exercise and $\mathrm{AD} .{ }^{35}$ Moreover, additional neuroimaging studies will be conducive to understanding the actual effect of exercise in patients at high risk of $\mathrm{AD}$.

\section{Acknowledgments}

This work was supported by the National Research Foundation of Korea (NRF) grant funded by the Korea government (MSIT) (No. 2019R1A2C2009100).

\section{Conflicts of Interest}

The authors have no potential conflicts of interest to disclose.

\section{Author Contributions}

Conceptualization: Yoo Hyun Um, Hyun Kook Lim. Data curation: Hyun Kook Lim. Formal analysis: Hyun Kook Lim. Funding acquisition: Hyun Kook Lim. Investigation: Sheng-Min Wang, Dong Woo Kang, Nak-Young Kim, Hae-Ran Na. Methodology: Hyun Kook Lim. Project administration: Hyun Kook Lim. Resources: Hyun Kook Lim. Supervision: Chang Uk Lee. Writing_original draft: Yoo Hyun Um. Writing_review\& editing: ShengMin Wang, Dong Woo Kang, Nak-Young Kim, Hae-Ran Na.

\section{ORCID iDs}

$\begin{array}{ll}\text { Yoo Hyun Um } & \text { https://orcid.org/0000-0002-3403-4140 } \\ \text { Sheng-Min Wang } & \text { https://orcid.org/0000-0003-2521-1413 } \\ \text { Nak-Young Kim } & \text { https://orcid.org/0000-0003-0116-6283 } \\ \text { Dong Woo Kang } & \text { https://orcid.org/0000-0003-3289-075X } \\ \text { Hae-Ran Na } & \text { https://orcid.org/0000-0002-7960-8603 } \\ \text { Chang Uk Lee } & \text { https://orcid.org/0000-0001-6398-7330 } \\ \text { Hyun Kook Lim } & \text { https://orcid.org/0000-0001-8742-3409 }\end{array}$

\section{REFERENCES}

1. Global, regional, and national burden of Alzheimer's disease and other dementias, 1990-2016: a systematic analysis for the Global Burden of Disease Study 2016. Lancet Neurol 2019;18:88-106.

2. Pereira CF, Santos AE, Moreira PI, Pereira AC, Sousa FJ, Cardoso SM, et al. Is Alzheimer's disease an inflammasomopathy? Ageing Res Rev 2019;56:100966.

3. Gong CX, Liu F, Iqbal K. Multifactorial hypothesis and multi-targets for Alzheimer's disease. J Alzheimers Dis 2018;64:S107-S117.

4. Armstrong RA. Risk factors for Alzheimer's disease. Folia Neuropathol 2019;57:87-105.

5. Kivipelto M, Mangialasche F, Ngandu T. Lifestyle interventions to pre- vent cognitive impairment, dementia and Alzheimer disease. Nat Rev Neurol 2018;14:653-666.

6. Na R, Kim YJ, Kim K, Kim KW. A systematic review of nonpharmacological interventions for moderate to severe dementia: a study protocol for a systematic review and meta-analysis. Psychiatry Investig 2018;15: 417-423.

7. Cass SP. Alzheimer's disease and exercise: a literature review. Curr Sports Med Rep 2017;16:19-22.

8. Bernardo TC, Marques-Aleixo I, Beleza J, Oliveira PJ, Ascensão A, Magalhães J. Physical exercise and brain mitochondrial fitness: the possible role against Alzheimer's disease. Brain Pathol 2016;26:648-663.

9. Hoffmann K, Sobol NA, Frederiksen KS, Beyer N, Vogel A, Vestergaard $\mathrm{K}$, et al. Moderate-to-high intensity physical exercise in patients with Alzheimer's disease: a randomized controlled trial. J Alzheimers Dis 2016;50:443-453.

10. Lamb SE, Mistry D, Alleyne S, Atherton N, Brown D, Copsey B, et al. Aerobic and strength training exercise programme for cognitive impairment in people with mild to moderate dementia: the DAPA RCT. Health Technol Assess 2018;22:1-202.

11. Cammisuli DM, Innocenti A, Fusi J, Franzoni F, Pruneti C. Aerobic exercise effects upon cognition in Alzheimer's disease: a systematic review of randomized controlled trials. Arch Ital Biol 2018;156:54-63.

12. Frederiksen KS, Gjerum L, Waldemar G, Hasselbalch SG. Effects of physical exercise on Alzheimer's disease biomarkers: a systematic review of intervention studies. J Alzheimers Dis 2018;61:359-372.

13. Schultz SA, Boots EA, Almeida RP, Oh JM, Einerson J, Korcarz CE, et al. Cardiorespiratory fitness attenuates the influence of amyloid on cognition. J Int Neuropsychol Soc 2015;21:841-850.

14. Williams VJ, Hayes JP, Forman DE, Salat DH, Sperling RA, Verfaellie M, et al. Cardiorespiratory fitness is differentially associated with cortical thickness in young and older adults. Neuroimage 2017;146:1084-1092.

15. Frederiksen KS, Larsen CT, Hasselbalch SG, Christensen AN, Høgh P, Wermuth L, et al. A 16-week aerobic exercise intervention does not affect hippocampal volume and cortical thickness in mild to moderate Alzheimer's disease. Front Aging Neurosci 2018;10:293.

16. Reiter K, Nielson KA, Smith TJ, Weiss LR, Alfini AJ, Smith JC. Improved cardiorespiratory fitness is associated with increased cortical thickness in mild cognitive impairment. J Int Neuropsychol Soc 2015;21:757-767.

17. de Oliveira Silva F, Ferreira JV, Plácido J, Sant’Anna P, Araújo J, Marinho $\mathrm{V}$, et al. Three months of multimodal training contributes to mobility and executive function in elderly individuals with mild cognitive impairment, but not in those with Alzheimer's disease: a randomized controlled trial. Maturitas 2019;126:28-33.

18. Morris JC. The Clinical Dementia Rating (CDR): current version and scoring rules. Neurology 1993;43:2412-2414.

19. Chun MY. Validity and reliability of Korean version of international physical activity questionnaire short form in the elderly. Korean J Fam Med 2012;33:144-151.

20. Lee JH, Lee KU, Lee DY, Kim KW, Jhoo JH, Kim JH, et al. Development of the Korean version of the Consortium to establish a Registry for Alzheimer's Disease Assessment Packet (CERAD-K): clinical and neuropsychological assessment batteries. J Gerontol B Psychol Sci Soc Sci 2002;57:47-53.

21. Park JH. Standardization of Korean version of the Mini-Mental State Examination (MMSE-K) for use in the elderly. Part II. Diagnostic validity. J Korean Neuropsychiatr Assoc 1989;28:508-513.

22. Daerr S, Brendel M, Zach C, Mille E, Schilling D, Zacherl MJ, et al. Evaluation of early-phase [18F]-florbetaben PET acquisition in clinical routine cases. NeuroImage Clin 2017;14:77-86.

23. Meltzer CC, Kinahan PE, Greer PJ, Nichols TE. Comparative evaluation of MR-based partial-volume correction schemes for PET. J Nucl Med 1999;40:2053-2065.

24. Koch K, Myers NE, Gottler J, Pasquini L, Grimmer T, Forster S, et al. Disrupted intrinsic networks link amyloid-beta pathology and impaired cognition in prodromal Alzheimer's disease. Cereb Cortex 2015;25: 
4678-4688.

25. Elman JA, Madison CM, Baker SL, Vogel JW, Marks SM, Crowley S, et al. Effects of beta-amyloid on resting state functional connectivity within and between networks reflect known patterns of regional vulnerability. Cereb Cortex 2016;26:695-707.

26. Dale AM, Fischl B, Sereno MI. Cortical surface-based analysis. I. Segmentation and surface reconstruction. Neuroimage 1999;9:179-194.

27. Fischl B, Dale AM. Measuring the thickness of the human cerebral cortex from magnetic resonance images. Proc Natl Acad Sci U S A 2000; 97:11050-11055.

28. Desikan RS, S?gonne F, Fischl B, Quinn BT, Dickerson BC, Blacker D, et al. An automated labeling system for subdividing the human cerebral cortex on MRI scans into gyral based regions of interest. Neuroimage 2006;31:968-980.

29. Fischl B, van der Kouwe A, Destrieux C, Halgren E, Ségonne F, Salat $\mathrm{DH}$, et al. Automatically parcellating the human cerebral cortex. Cereb Cortex 2004;14:11-22.

30. Fischl B, Sereno MI, Dale AM. Cortical surface-based analysis. II: Inflation, flattening, and a surface-based coordinate system. Neuroimage 1999;9:195-207.

31. Loprinzi PD, Edwards MK, Crush E, Ikuta T, Del Arco A. Dose-response association between physical activity and cognitive function in a national sample of older adults. Am J Health Promot 2018;32:554-560.

32. Fondell E, Townsend MK, Unger LD, Okereke OI, Grodstein F, Ascherio $\mathrm{A}$, et al. Physical activity across adulthood and subjective cognitive function in older men. Eur J Epidemiol 2018;33:79-87.

33. Frith E, Loprinzi PD. Physical activity is associated with higher cognitive function among adults at risk for Alzheimer's disease. Complement Ther Med 2018;36:46-49.

34. Loprinzi PD, Frith E. Association between perceived physical activity and cognitive function in older adults. Psychol Rep 2019;122:108-116.

35. Falck RS, Davis JC, Best JR, Crockett RA, Liu-Ambrose T. Impact of exercise training on physical and cognitive function among older adults: a systematic review and meta-analysis. Neurobiol Aging 2019;79:119-130.

36. Voss MW, Erickson KI, Prakash RS, Chaddock L, Kim JS, Alves H, et al. Neurobiological markers of exercise-related brain plasticity in older adults. Brain Behav Immun 2013;28:90-99.

37. Håkansson K, Ledreux A, Daffner K, Terjestam Y, Bergman P, Carlsson $\mathrm{R}$, et al. BDNF responses in healthy older persons to 35 minutes of physical exercise, cognitive training, and mindfulness: associations with working memory function. J Alzheimers Dis 2017;55:645-657.

38. Chupel MU, Minuzzi LG, Furtado G, Santos ML, Hogervorst E, Filaire E, et al. Exercise and taurine in inflammation, cognition, and peripheral markers of blood-brain barrier integrity in older women. Appl Physiol
Nutr Metab 2018;43:733-741.

39. van der Kleij LA, Petersen ET, Siebner HR, Hendrikse J, Frederiksen KS, Sobol NA, et al. The effect of physical exercise on cerebral blood flow in Alzheimer's disease. Neuroimage Clin 2018;20:650-654.

40. Ferrari L, Vicenzi M, Tarantini L, Barretta F, Sironi S, Baccarelli AA, et al. Effects of physical exercise on endothelial function and DNA methylation. Int J Environ Res Public Health 2019;16:2530.

41. Hu ML, Zong XF, Mann JJ, Zheng JJ, Liao YH, Li ZC, et al. A review of the functional and anatomical default mode network in schizophrenia. Neurosci Bull 2017;33:73-84.

42. Chirles TJ, Reiter K, Weiss LR, Alfini AJ, Nielson KA, Smith JC. Exercise training and functional connectivity changes in mild cognitive impairment and healthy elders. J Alzheimers Dis 2017;57:845-856.

43. Zidan M, Boban J, Bjelan M, Todorović A, Stankov Vujanić T, Semnic $\mathrm{M}$, et al. Thalamic volume loss as an early sign of amnestic mild cognitive impairment. J Clin Neurosci 2019;68:168-173.

44. Tentolouris-Piperas V, Ryan NS, Thomas DL, Kinnunen KM. Brain imaging evidence of early involvement of subcortical regions in familial and sporadic Alzheimer's disease. Brain Res 2017;1655:23-32.

45. Yi HA, Möller C, Dieleman N, Bouwman FH, Barkhof F, Scheltens P, et al. Relation between subcortical grey matter atrophy and conversion from mild cognitive impairment to Alzheimer's disease. J Neurol Neurosurg Psychiatry 2016;87:425-432.

46. Yamamoto M, Wada-Isoe K, Yamashita F, Nakashita S, Kishi M, Tana$\mathrm{ka} \mathrm{K}$, et al. Association between exercise habits and subcortical gray matter volumes in healthy elderly people: a population-based study in Japan. eNeurologicalSci 2017;7:1-6.

47. Tarazona-Santabalbina FJ, Gómez-Cabrera MC, Pérez-Ros P, Martínez-Arnau FM, Cabo H, Tsaparas K, et al. A multicomponent exercise intervention that reverses frailty and improves cognition, emotion, and social networking in the community-dwelling frail elderly: a randomized clinical trial. J Am Med Dir Assoc 2016;17:426-433.

48. Northey JM, Cherbuin N, Pumpa KL, Smee DJ, Rattray B. Exercise interventions for cognitive function in adults older than 50: a systematic review with meta-analysis. Br J Sports Med 2018;52:154-160.

49. Dedeyne L, Deschodt M, Verschueren S, Tournoy J, Gielen E. Effects of multi-domain interventions in (pre)frail elderly on frailty, functional, and cognitive status: a systematic review. Clin Interv Aging 2017;12: 873-896.

50. Imaoka M, Nakao H, Nakamura M, Tazaki F, Maebuchi M, Ibuki M, et al. Effect of multicomponent exercise and nutrition support on the cognitive function of older adults: a randomized controlled trial. Clin Interv Aging 2019;14:2145-2153. 